\title{
Damage evolution and fracture of ceramic coating systems in circle plate bending tests: Experimental observation and modeling
}

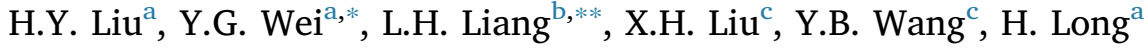

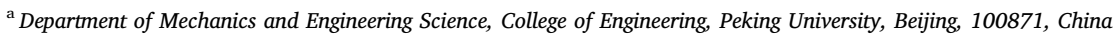 \\ ${ }^{\mathrm{b}}$ College of Mechanical and Electrical Engineering, Beijing University of Chemical Technology, Beijing, 100029, China \\ ${ }^{\mathrm{c}}$ LNM, Institute of Mechanics, Chinese Academy of Sciences, Beijing, 100190, China
}

\section{A R T I C L E I N F O}

\section{Keywords:}

Ceramic coating systems

Fracture

Damage model

Circle plate bending

\begin{abstract}
A B S T R A C T
The coating thickness-dependent damage and fracture of ceramic coating systems were investigated systematically by using circle plate bending tests based on a scanning electron microscope. Through experimental observations and measurements, results demonstrated that the damage of coating layer appeared because of effects of both radial and circumferential moments for thin coating cases, and because of main circumferential moment effect for thick ones. A circle plate bending damage model for the coating layer was presented to describe coating layer damage evolution and fracture. Via the model, one can effectively predict the failure phenomena of coating layer under the circle plate bending, which were consistent with experimental observations. In addition, modeling results of the relationships between critical external loads and damage zone sizes for coating layer were also consistent with experimental measurements. The damage was found to have a catastrophic characteristic near the failure point based on the mathematic damage model.
\end{abstract}

\section{Introduction}

Ceramic coating systems are widely used in many engineering fields, such as aerospace, mechanical, and chemical engineering, because of its excellent characteristics in resisting the thermal shock, erosion and wear, etc. [1-5]. For example, thermal barrier coatings (TBCs) with low thermal conductivity provide excellent thermal protection and have been widely used in blade thermal protection of higher-temperature turbines. The damage, spalling, and fracture of ceramic coatings lead to the Ni-based super-alloy substrate getting exposed to the high-temperature environment and thus failing to work. Therefore, it is important to investigate the damage and fracture resistance characteristics of ceramic coating systems, and it is helpful to predict the lifetimes of coating systems.

Both transverse cracking and interfacial delamination in ceramic coating systems are the typical damage and fracture modes observed when the systems are exposed to extreme service conditions such as oxidation [6-9], thermal cycles [10-14], and thermal-mechanical coupling. In order to study the damage and fracture mechanism for the coating systems, several test and numerical simulation methods have been presented and widely used, such as tensile tests [15-18], beam bending tests [19-24], indentation tests [25-28], and several numerical simulation methods [29-31]. These experiments and simulations can synchronously monitor the propagation process of damage and fracture. In the past two decades, several studies have been conducted and have shown that the fracture modes of coating systems depend on many factors, such as the processing method, coating thickness, and loading and heat treatment conditions. Among these factors, the effect of coating thickness on damage and fracture behavior of the systems has been the subject of many researches, according to literatures [23-26,30,31]. By using the three-point bending tests, Li et al. found that transverse cracking dominated in thin coating systems, whereas interface delamination dominated in thick ones [23], which is consistent with literature [24]. By using the indentation test method, He et al. found, theoretically and experimentally, that the fracture modes of brittle coatings depended on the coating thickness [26]. Zhu et al. determined, through tensile simulation, that surface crack density and interface crack length depended on coating thickness [30]. Their results have shown that the thinner the coating thickness, the larger the surface crack density, and the shorter the length of the interface cracks. Xu et al. discovered, via numerical simulation, that the thickness ratio of two ceramic layers had an effect on the failure mechanisms of a thermal barrier coating system with double ceramic layers [31]. They found that a thicker outermost ceramic layer made the delamination in the

\footnotetext{
* Corresponding author.

** Corresponding author.

E-mail address: weiyg@pku.edu.cn (Y.G. Wei).
} 
interface between two ceramic coatings more likely to occur.

The damage evolution (process of crack propagation) of coating systems have been studied further using statistics [15,32-36] and damage models [37-42]. These statistical analyses include counting the change in the number (destiny) or area of the cracks throughout the whole loading process. Zhang et al. calculated the variation of cracking density with respect to the applied tensile strain under four-point bending tests [33]. Schweda et al. investigated the variation of the interface crack area with respect to the number of thermal oxidation cycles [35]. Qian et al. calculated the variation of the number of transverse cracks with respect to tensile strain and proposed a damage evolution model for sandwiched coating systems under tensile loading [37]. The initiation, propagation, and saturation of transverse cracks in the coatings were modeled. Hille et al. proposed a numerical damage model to describe the development of the thermally grown oxide map and the fracture processes in thermal barrier coating systems [39]. Yang et al. used a modified shear-lag model to calculate the surface crack density in thermal barrier coatings [40] and found that it was in agreement with the result obtained using acoustic emission and digital image correlation methods. Saucedo-Mora et al. developed a multiscale fracture model to simulate thermo-mechanical damage in a thermal barrier coating system [41]. Liang et al. proposed a catastrophic failure damage model based on mathematical analysis [42,43]. They investigated the crack evolution behavior of the coating systems under three- and four-point bending tests and found that it had catastrophic failure characteristics similar to those found in bulk brittle materials [44].

In order to investigate the catastrophic failure characteristics for the coating layer in the coating/Ni-based super-alloy substrate system in our research, we will present a circle plate bending test method, investigate the accompanying catastrophic behavior via the damage evolution and fracture within the coating layer, and investigate the size effects of coating layer thickness on the mechanical behavior of the coating/substrate system.

In this study, circle plate bending tests are performed and crack evolution is observed with a scanning electron microscope (SEM) to determine the damage and fracture features of coating systems with different coating thicknesses. The damage and fracture characteristics are investigated by observing the crack maps, and a theoretical model is proposed to describe the coating thickness-dependent failure process of the coating/substrate systems. The damage evolution behavior of the coating layer with respect to applied load is studied. In addition, for comparison with our previous three-point and four-point bending experimental results [42,43], a mathematical damage model is also used to describe the damage evolution process for the circle plate bending experiments.

\section{Circle plate bending experiments for coating layer fracture}

\subsection{Experimental procedure}

Fig. 1 shows the circle plate bending test method we designed in this research. The experimental samples were multilayer structures, consisting of substrates, bond coats, and ceramic coating layers. A Ni-based

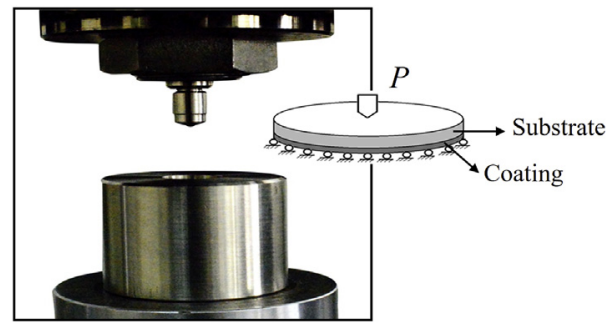

Fig. 1. Geometry and principle of circle plate bending test.
Table 1

Thicknesses of coatings, elastic limit loads, failure loads, normalized initial damage, normalized initial load, and damage coefficient.

\begin{tabular}{lllllll}
\hline Sample symbol & $h_{1}(\mu \mathrm{m})$ & $P_{e}(\mathrm{~N})$ & $P_{f}(\mathrm{~N})$ & $D_{0}$ & $\lambda_{0}$ & $C$ \\
\hline M1 & 70 & 1000 & 2700 & 0.08 & 0.37 & 1.16 \\
M2 & 180 & 1000 & 2500 & 0.08 & 0.40 & 1.18 \\
M3 & 400 & 1000 & 2500 & 0.06 & 0.40 & 1.21 \\
\hline
\end{tabular}

super alloy (GH3128) with a thickness of $1.2 \mathrm{~mm}\left(h_{2}\right)$ was used as the substrate material. NiCrAlY bond coat with a thickness of approximately $10 \mu \mathrm{m}$ was sprayed on the substrate. Then $8 \mathrm{wt} \% \mathrm{Y}_{2} \mathrm{O}_{3}$-stabilized $\mathrm{ZrO}_{2}$ top coat was sprayed on the bond coat. We used the standard air plasma spray method to prepare the samples, as shown in previous work [45]. The samples for the bending experiments were circle plates with polished coating surface and diameters of $14 \mathrm{~mm}$. The radius $a$ of the plate under load was $4 \mathrm{~mm}$, and the diameter of the Brinell indenter was $1 \mathrm{~mm}$. Three kinds of coating thickness samples were used to study the thickness-dependent fracture and damage characteristics.

Circle plate bending experiments were performed at room temperature and a constant displacement loading rate of $0.1 \mathrm{~mm} / \mathrm{min}$. The load and displacement were controlled by the testing machine (MTS 810); the load was applied to the upper surface of the alloy substrates so that the coating layer was in the tensile stress state, as shown in Fig. 1. Three representative samples with different coating-layer thicknesses $\left(h_{1}\right)$, as shown in Table 1 , were used in the first experiment. The experiment was interrupted and unloaded at a series of different loading levels. The samples were placed under SEM to observe the crack maps of the coating surface after unloading each time, and then the samples continued to be reloaded; this step was repeated until the load reached $3000 \mathrm{~N}$. Nine loading levels were chosen according to multiple experiments to obtain more details of crack evolution. In the second experiment, another three representative samples, as shown in Table 1 , were directly loaded with $3000 \mathrm{~N}$, and then the samples were placed under SEM to observe the crack maps of the coating surface to study the thickness dependence of the fracture modes.

\subsection{Experimental measurement and observation}

Fig. 2 shows the load-displacement curves for the three representative samples M1, M2, and M3 (circularly loaded to $3000 \mathrm{~N}$ in the first circle plate bending tests). From the figure, it can be seen that the thicker coating systems demonstrated larger loads than the thinner ones at the same displacement. Note that some hysteresis loops in the

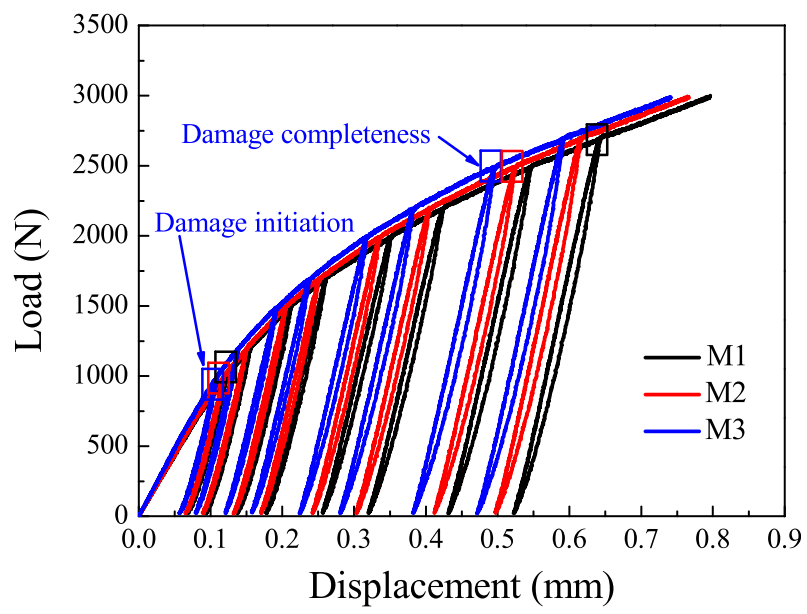

Fig. 2. Load-displacement curves of the representative samples M1, M2, and M3 (circularly loaded to $3000 \mathrm{~N}$ in the first circle plate bending tests). The region between the two squares is the damage step. 


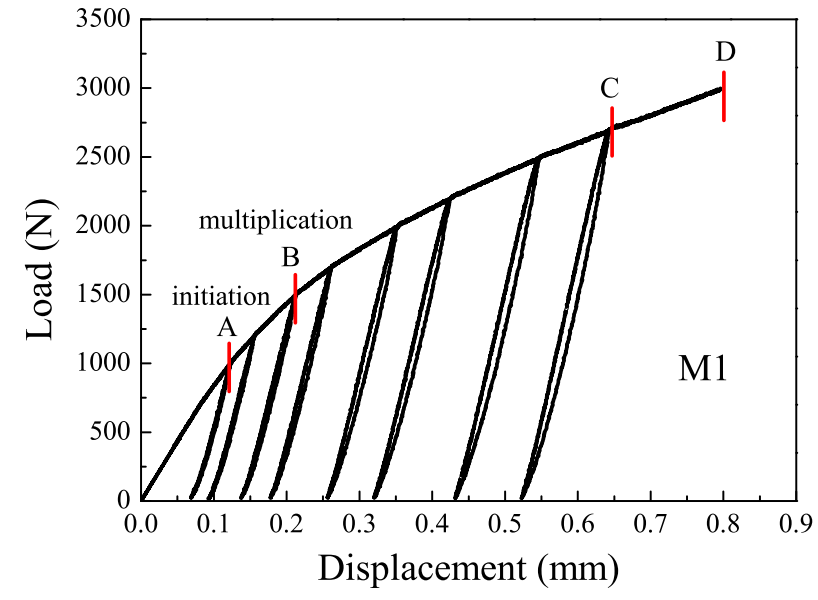

Fig. 3. Load-displacement curve of the thinnest coating system M1 (circularly loaded to $3000 \mathrm{~N}$ in the first circle plate bending tests). The short lines denote the four loading points (A-D). The corresponding maps are given in Figs. 4a-d.

curves corresponded to the points at which the experiment was interrupted and unloaded, and then reloaded again after the crack maps were captured under SEM. The parts between the two squares in the curves show the damage steps. The detailed selection of the damage step is discussed in the next section.

For a more obvious contrast of damage evolution behaviors and fracture characteristics, the load-displacement curve of the thinnest coating system (M1) in Fig. 3 and the corresponding crack evolution maps in Fig. 4 were compared with the load-displacement curve of the thickest one (M3) in Fig. 5 and the corresponding crack evolution maps in Fig. 6. The crack maps with a noticeable change were chosen for display, as shown in Fig. 4 and Fig. 6, for a clearer demonstration of crack evolution behaviors. For the thin coating system M1, both the radial and circumferential cracks initiated in the coating layer at a load of $1000 \mathrm{~N}$, corresponding to point $\mathrm{A}$ on the curve in Fig. 3 (see Fig. 4(a)). The radial and circumferential cracks propagated and multiplied at a load of $1500 \mathrm{~N}$, corresponding to point $\mathrm{B}$ on the curve in Fig. 3 (see Fig. 4(b)). The multiple radial and circumferential cracks

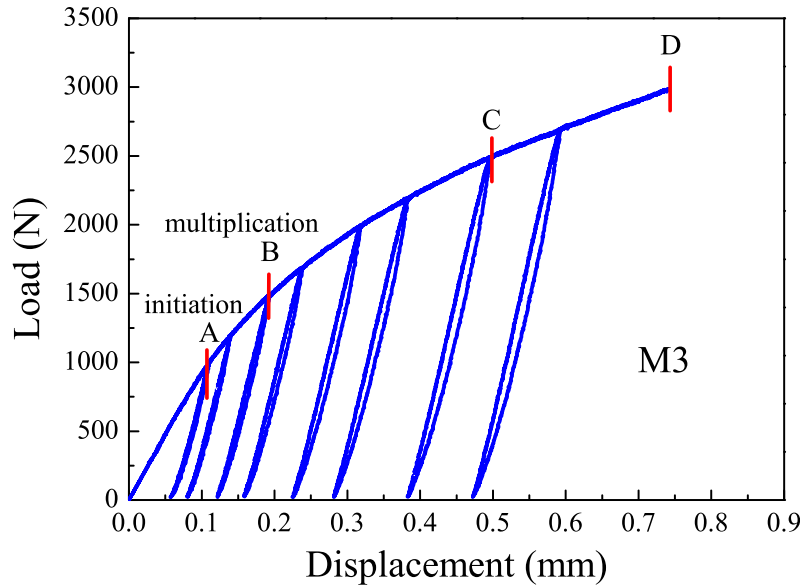

Fig. 5. Load-displacement curve of the thickest coating system M3 (circularly loaded to $3000 \mathrm{~N}$ in the first circle plate bending tests). The short lines denote the four loading points (A-D). The corresponding maps are given in Figs. 6a-d.

proceeded to saturation when the load reached about $2000 \mathrm{~N}$. After saturation, the radial and circumferential cracks became wider and crack density was kept unchangeable, as shown in Fig. 3 (and Fig. 4(c)) corresponding to load $2700 \mathrm{~N}$ at point $\mathrm{C}$, and in Fig. 3 (and Fig. 4(d)) corresponding to load $3000 \mathrm{~N}$ at point $\mathrm{D}$. The crack evolution behavior of the thick coating system (M3) is similar to that of the thin one (M1), while mainly the radial cracking occurred. In the thick coating system, it can be seen from Fig. 6 that, the radial crack evolution includes initiation (Fig. 6(a)), multiplication (Fig. 6(b)), and saturation at load also about $2000 \mathrm{~N}$. After saturation, the radial cracks becomes wider and crack density is kept unchangeable approximately, as seen in Fig. 5 (and Fig. 6(c)) corresponding to load $2500 \mathrm{~N}$ at point $\mathrm{C}$, and in Fig. 5 (and Fig. 6(d)) corresponding to load $3000 \mathrm{~N}$ at point $\mathrm{D}$.

\subsection{Thickness-dependent damage and fracture}

Fig. 7 shows the measured load-displacement curves for the three representative samples M1, M2, and M3 (directly loaded with $3000 \mathrm{~N}$ in
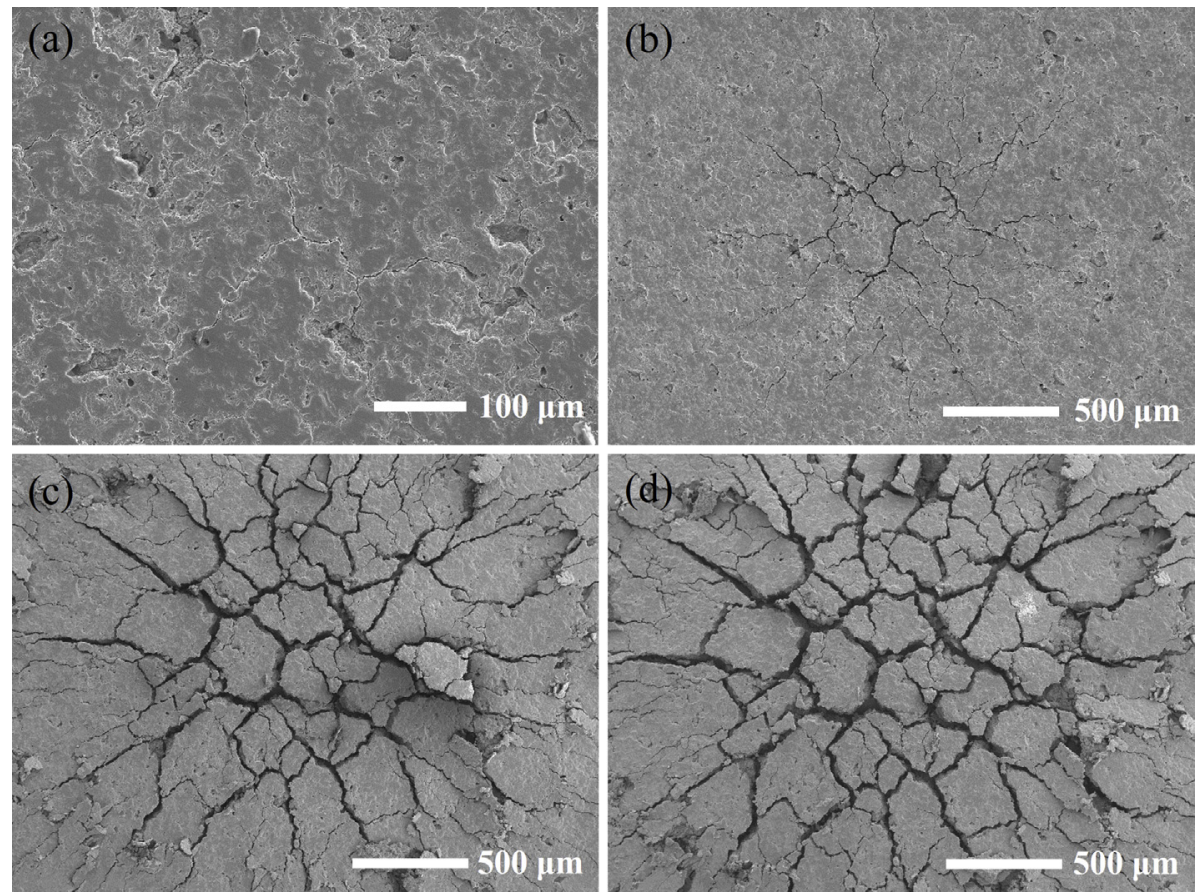

Fig. 4. Crack maps of the coating surface for M1 at the loading levels of points A-D in Fig. 3. 

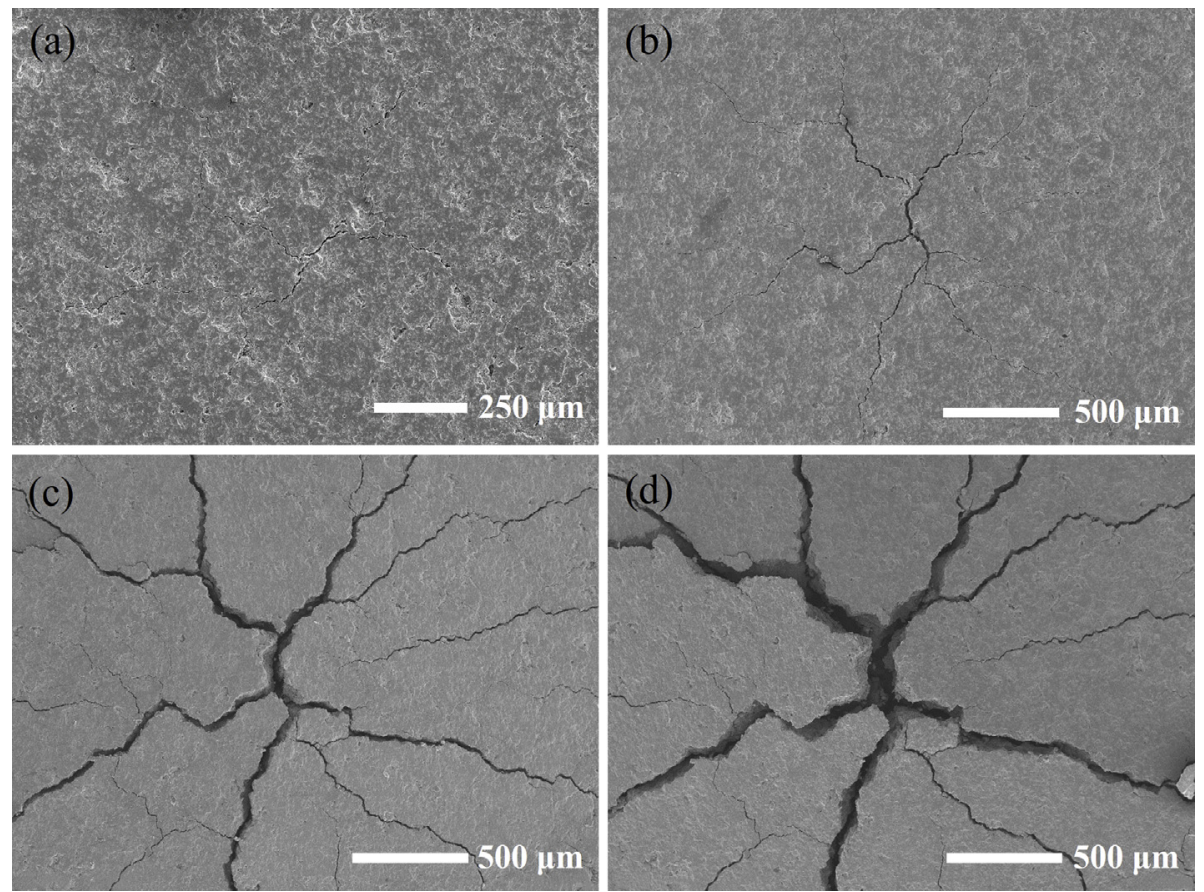

Fig. 6. Crack maps of the coating surface for M3 at the loading levels of points A-D in Fig. 5.

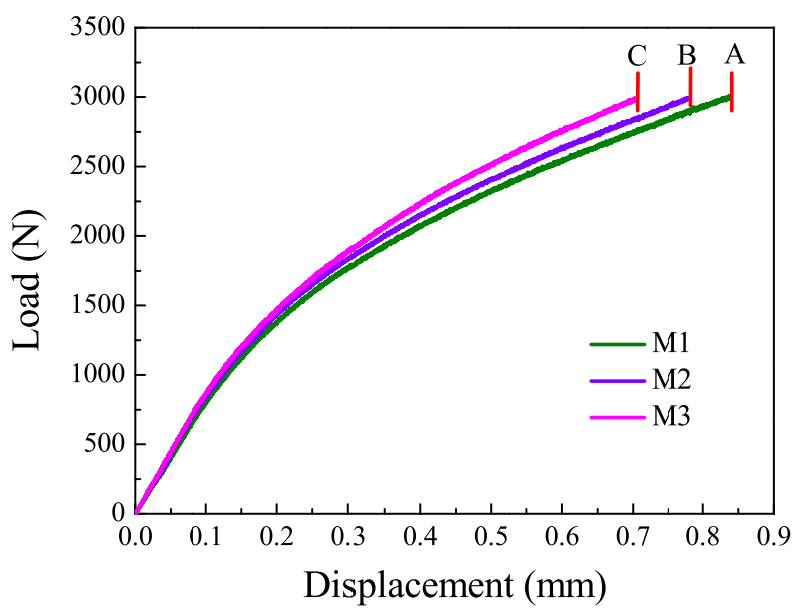

Fig. 7. Load-displacement curves of the representative samples M1, M2, and M3 (directly loaded to $3000 \mathrm{~N}$ in the second circle plate bending tests). The short lines denote the final loading points (A-C), and the corresponding crack maps are given in Figs. 8a-c. The load-displacement curves should be cut off at about $P=2000$ Nif they are predicted by the small-deflection bending model.

the second circle plate bending tests). Comparing with Fig. 2, one can find that the load-displacement curves based on the monotonically loading process shown in Fig. 7 are consistent with the outlines of circularly loading cases shown in Fig. 2; therefore, the effect of unloading on the outline of the load-displacement curve can be ignored. The thicker the coating layer thickness, the smaller the load and slope of the linear section. The main reason is that the thicker coating layer in the system had a greater equivalent bending stiffness, based on the bending theory of composite plates. Fig. 8 shows the crack maps for M1, $\mathrm{M} 2$, and M3 at the final loading levels (A-C). The three kinds of coating systems all fractured by tensile loading within the coating layer. In the thin coating systems (M1-M2), the fractures were dominated by radial and circumferential moments, whereas in the thick coating system (M3), the fracture was dominated mainly by the circumferential moment. The radial and circumferential crack number decreased with increasing coating layer thickness. When the coating layer thickness reached a certain value, mainly the radial cracking occurred in the coating layer, as shown in Fig. 8(c).

It is worth noting that the features of crack maps in Fig. 4 (c) and (d), Fig. 6 (c) and (d), as well as Fig. 8 are similar to those when load level is about $2000 \mathrm{~N}$, at which the crack density has reached at saturation, although the deflection is small.

\section{Modeling of the thickness-dependent damage and fracture for coating layer}

In order to describe the thickness-dependent damage evolution behaviors and fracture characteristics of the coating/substrate systems, a
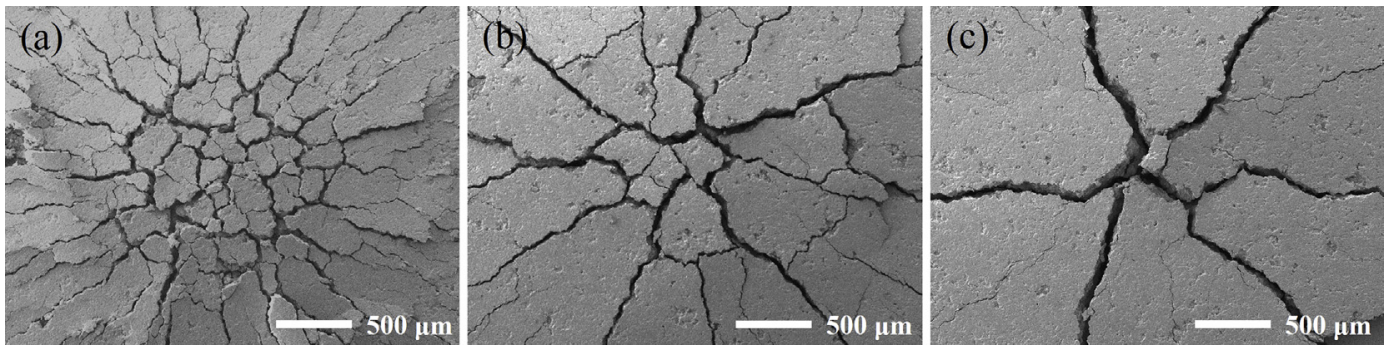

Fig. 8. Crack maps of the coating surface under the indenter at the final loading levels of points A-C in Fig. 7. The thicknesses of coating layer from (a) to (c) are $70 \mu \mathrm{m}, 180 \mu \mathrm{m}$, and $400 \mu \mathrm{m}$, respectively. 
theoretical model considering a circular fully damaged region within the coating layer was proposed to predict the thickness-dependent damage and fracture. In addition, for comparison, the previous mathematical damage model $[42,43]$ was also used here to describe the damage evolution behavior.

\subsection{Theoretical model considering a circular fully damaged region in coating layer}

Considering that the bond coat layer thickness is much thinner than the ceramic coating layer and substrate, and the fundamental material parameters of bond coat layer, such as Young's modulus, Poisson's ratio, mass density as well as thermal conductivity, are close to those of substrate [46], therefore, in the present model the coating/substrate system is simply treated as a two-layer plate bending model, and the bond coat layer is incorporated as the substrate. The indenter loading is treated simply as a concentrated load $P$, as sketched in Fig. 1 . We take the critical value of the effective moment $M_{e}=M_{c}$ in the coating/substrate system as the limit condition for the coating layer cracking in the axisymmetric circular plate bending:

$M_{\theta}^{2}-M_{\theta} M_{r}+M_{r}^{2}=M_{c}^{2}$,

where $M_{r}$ and $M_{\theta}$ are the radial and circumferential bending moments, respectively, and $M_{c}$ is the critical effective bending moment. We can define a fracture function for the brittle fracture of coating layer based on Eq. (1):

$f(r, \quad P)=\left(\frac{M_{\theta}}{M_{c}}\right)^{2}-\frac{M_{\theta}}{M_{c}} \frac{M_{r}}{M_{c}}+\left(\frac{M_{r}}{M_{c}}\right)^{2}-1 \leq 0$,

where $r$ is the radial coordinate from the center of the circular plate.

Since we mainly concern with the loading-displacement relations during coating layer failure process, so in the analysis model we can always use the small-deflection bending theory to describe the deformation and failure process for the coating layer/substrate system. However, the reliability of the prediction results should be cut off if the system gets into a finite deformation when applied load is large. In the present research, the predictions based on the small-deflection bending theory can be taken as a reference to the experimental results for the finite deformation case.

As we know, the effective moment at the center of the plate first reaches the critical value when an exerted concentrated load reaches the critical load. Therefore, one can imagine that there exists a damage zone $r \leq r_{c}=b$ within the coating layer around the center point of the coating/substrate plate, where $r_{c}$ is the critical cracking radius, and $b$ is the damage zone size, therefore, $r_{c}$ is always equal to $b$. When $r=r_{c}=b$, Eq. (1) is satisfied, and Eq. (2) indicates that the radius corresponding to the maximum fracture function $r=r_{c}=b$ is the cracking front. As load $P$ continuously increases, the damage zone size $b$ increases, so that the theoretical model we present here is described by a circle composite plate bending geometry with a circular damaged region in coating layer, as shown in Fig. 9. In the following analysis in the subsection, we use the theoretical model to approximately describe the damage evolution of the coating layer.

In the circular plate bending geometry shown in Fig. 9, $b$ is the

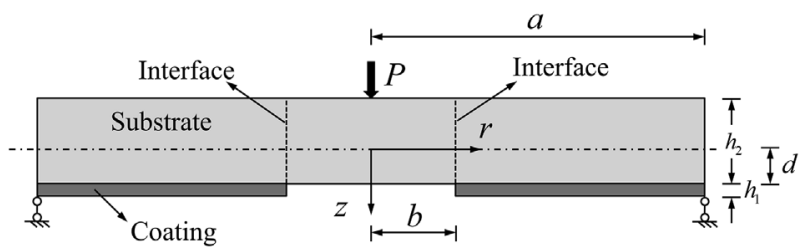

Fig. 9. Circle coating/substrate plate bending model with a circular fully damaged region in coating layer. Front of full damage zone is at $r=r_{c}=b$, where effective moment arrives at the critical value. radius of cracking zone, and the substrate in the coating cracking zone is regarded as a thin plate, the center of the plate is subjected to a concentrated load $P$, and the edge of the plate is restricted by the interface. The coating/substrate system in the undamaged coating zone is regarded as a composite thin plate part, and the distance between the coating/substrate interface and the neutral plane of the composite plate is denoted as $d$. The outer radius of the plate is denoted as $a$, the edge of the plate is treated approximately as a simple support, and the edge of the hole (in substrate) is treated as satisfying the continuity conditions of deflection, rotation angle, and moments (as seen in following Eq. (14)).

The deflection solutions of the circle plate bending are denoted as $w^{(1)}$ and $w^{(2)}$, respectively, within the regions $0 \leq r \leq b, b \leq r \leq a$. The equilibrium differential equation expressed by deflection is as follows:

$\nabla^{2} \nabla^{2} w^{(i)}=0, \quad(i=1,2)$,

where $\nabla^{2}=\frac{\partial^{2}}{\partial r^{2}}+\frac{1}{r} \frac{\partial}{\partial r}$. The general solutions of deflections are

$w^{(1)}=c_{1} \ln r+c_{2} r^{2} \ln r+c_{3} r^{2}+c_{4} \quad(0 \leq r \leq b)$,

$w^{(2)}=c_{5} \ln r+c_{6} r^{2} \ln r+c_{7} r^{2}+c_{8} \quad(b \leq r \leq a)$,

where the coefficients $c_{1}-c_{8}$ are undetermined constants. According to the small deflection bending theory, the radial and circumferential bending moments and the shear force in the $z$ direction of the thin plate bending can be expressed respectively as

$M_{r}^{(i)}=-\left(S_{11}^{(i)} \frac{\mathrm{d}^{2} w^{(i)}}{\mathrm{d} r^{2}}+\frac{S_{12}^{(i)}}{r} \frac{\mathrm{d} w^{(i)}}{\mathrm{d} r}\right)$,

$M_{\theta}^{(i)}=-\left(S_{21}^{(i)} \frac{\mathrm{d}^{2} w^{(i)}}{\mathrm{d} r^{2}}+\frac{S_{22}^{(i)}}{r} \frac{\mathrm{d} w^{(i)}}{\mathrm{d} r}\right)$,

$Q_{r z}^{(i)}=\frac{\mathrm{d} M_{r}^{(i)}}{\mathrm{d} r}+\frac{M_{r}^{(i)}-M_{\theta}^{(i)}}{r}=-S_{11}^{(i)}\left(\frac{\mathrm{d}^{3} w^{(i)}}{\mathrm{d} r^{3}}+\frac{1}{r} \frac{\mathrm{d}^{2} w^{(i)}}{\mathrm{d} r^{2}}-\frac{1}{r^{2}} \frac{\mathrm{d} w^{(i)}}{\mathrm{d} r}\right)$,

where the superscripts $(i=1,2)$ denote the solutions of two regions at $0 \leq r \leq b, b \leq r \leq a$, respectively. $S_{11}$ and $S_{12}$ are the bending stiffness of the thin plate, $S_{22}^{(1)}=S_{11}^{(1)}=\frac{E_{2} h_{2}^{3}}{12\left(1-v_{2}^{2}\right)}$, and $S_{21}^{(1)}=S_{12}^{(1)}=\nu_{2} S_{11}^{(1)}$ for the first region $0 \leq r \leq b$. Specifically, the bending stiffness within the second region $b \leq r \leq a$ can be written as

$S_{22}^{(2)}=S_{11}^{(2)}=\frac{1}{1-v_{1}^{2}} \frac{E_{1}}{3}\left[\left(h_{1}+d\right)^{3}-d^{3}\right]+\frac{1}{1-v_{2}^{2}} \frac{E_{2}}{3}\left[\left(h_{2}-d\right)^{3}+d^{3}\right]$,

$S_{21}^{(2)}=S_{12}^{(2)}=\frac{\nu_{1}}{1-v_{1}^{2}} \frac{E_{1}}{3}\left[\left(h_{1}+d\right)^{3}-d^{3}\right]+\frac{\nu_{2}}{1-v_{2}^{2}} \frac{E_{2}}{3}\left[\left(h_{2}-d\right)^{3}+d^{3}\right]$,

where $d=\frac{E_{2} h_{2}^{2}-E_{1} h_{1}^{2}}{2\left(E_{2} h_{2}+E_{1} h_{1}\right)}$ is the distance from the neutral plane to the interface based on the small deflection bending theory for the coating/ substrate composite thin plate.

The coefficients $c_{1}-c_{8}$ in deflection functions Eqs. (4) and (5) can be determined by using the following conditions.

Symmetry condition:

$\left.\frac{\mathrm{d} w^{(1)}}{\mathrm{d} r}\right|_{r=0}=0$

Equilibrium conditions:

$2 \pi r Q_{r z}^{(i)}=-P \quad(i=1,2)$

Boundary conditions: 
$\left.w^{(2)}\right|_{r=a}=0,\left.\quad M_{r}^{(2)}\right|_{r=a}=0$

Continuity conditions:

$\left.w^{(1)}\right|_{r=b}=\left.w^{(2)}\right|_{r=b},\left.\quad \frac{\mathrm{d} w^{(1)}}{\mathrm{d} r}\right|_{r=b}=\left.\frac{\mathrm{d} w^{(2)}}{\mathrm{d} r}\right|_{r=b},\left.\quad M_{r}^{(1)}\right|_{r=b}=\left.M_{r}^{(2)}\right|_{r=b}$

We can determine the coefficients $c_{1}-c_{8}$ in deflection expressions by using conditions (11)-(14). The results are shown in Appendix A.

Through the use of Eqs. (5)-(7), the radial and circumferential bending moments within the region $b \leq r \leq a$ can be given:

$M_{r}^{(2)}=-S_{11}^{(2)}\left\{\frac{\eta-1}{r^{2}} c_{5}+[2(1+\eta) \ln r+(3+\eta)] c_{6}+2(1+\eta) c_{7}\right\}$,

$M_{\theta}^{(2)}=-S_{11}^{(2)}\left\{\frac{1-\eta}{r^{2}} c_{5}+[2(1+\eta) \ln r+(1+3 \eta)] c_{6}+2(1+\eta) c_{7}\right\}$,

where $\eta=\frac{S_{12}^{(2)}}{S_{11}^{(2)}}$.

When Eqs. (15)-(16) are combined, the fracture function Eq. (2) can be finally written as

$f(r, P)=\left(\frac{P}{8 \pi M_{c}}\right)^{2} f_{1}(r)-1 \leq 0$,

where

$f_{1}(r)=\left(\frac{S_{11}^{(2)}}{S_{11}^{(1)}}\right)^{2}\left[\mathrm{~A}(r)^{2}-\mathrm{A}(r) * \mathrm{~B}(r)+\mathrm{B}(r)^{2}\right] \quad(b \leq r \leq a)$,

and

$\mathrm{A}(r)=\frac{1-\eta}{r^{2}} \frac{c_{5}}{c_{2}}+[2(1+\eta) \ln r+(1+3 \eta)] \frac{c_{6}}{c_{2}}+2(1+\eta) \frac{c_{7}}{c_{2}}$,

$\mathrm{B}(r)=\frac{\eta-1}{r^{2}} \frac{c_{5}}{c_{2}}+[2(1+\eta) \ln r+(3+\eta)] \frac{c_{6}}{c_{2}}+2(1+\eta) \frac{c_{7}}{c_{2}}$.

For the coating/substrate system used in experiments, the substrate thickness and the plate radius can be measured and are constant ( $h_{2}=1.2 \mathrm{~mm}, a=4 \mathrm{~mm}$ ), whereas the thickness of the coating $h_{1}$ is adjusted (as seen in Table 1). The other material parameters are taken as $E_{1}=100 \mathrm{GPa}, E_{2}=200 \mathrm{GPa}, \nu_{1}=0.1$, and $\nu_{2}=0.3$. Substituting the material and geometric parameters into Eq. (18), one can obtain the relationship of function $f_{1}(r)$ with $r$, as shown in Fig. 10 for $b=1 \mathrm{~mm}$. It can be seen from Fig. 10 that the function $f_{1}(r)$ in the domain of definition $(b \leq r \leq a)$ decreases monotonically as $r$ increases, and the

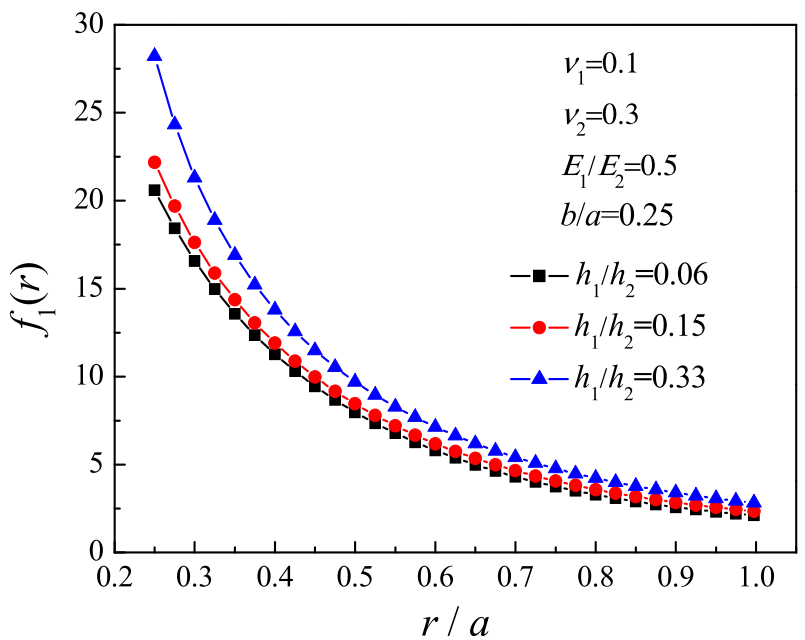

Fig. 10. Function $f_{1}(r)$ versus radius $r$ at $b=1 \mathrm{~mm}$ for the three kinds of coating systems.

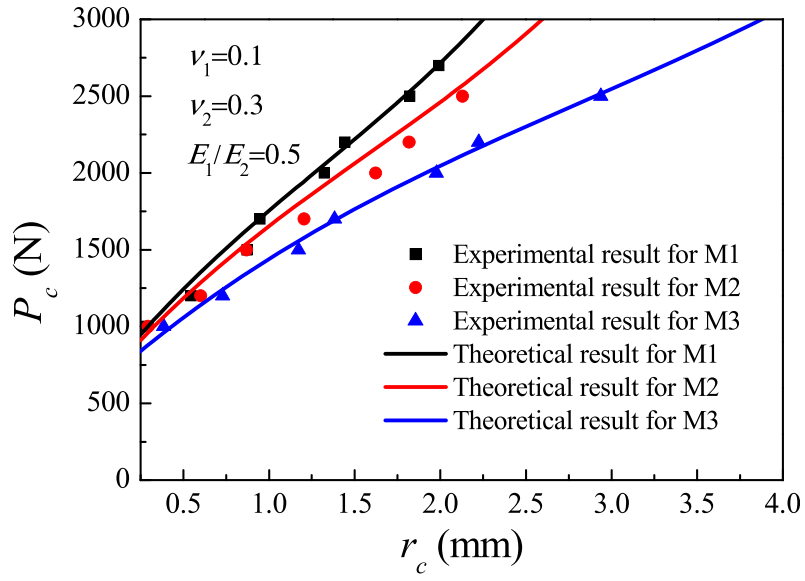

Fig. 11. Critical cracking load versus critical cracking radius for the three kinds of coating systems M1, M2, and M3 (circularly loaded to $3000 \mathrm{~N}$ in the first circle plate bending tests). The symbols are the experimental data, and the curves are based on theoretical model.

function maximizes at $r=b$; therefore, the cracks always propagates outward with load increase.

From Eq. (17), one can obtain the critical load of coating layer cracking by letting $f(r, P)=0$ at $r=b$ :

$P_{c}=\frac{8 \pi M_{c}}{\sqrt{f_{1}(b)}}$

where $M_{c}$ is the critical effective bending moment, which is the material parameter and can be measured via circle plate bending test based on Eq. (1); as in this research, the crack maps were observed in the experiments (as seen in Figs. 4 and 6). The values of $M_{c}$ measured are $313.88 \mathrm{~N}, 306.71 \mathrm{~N}$, and $304.30 \mathrm{~N}$ for samples M1, M2, and M3 (circularly loaded to $3000 \mathrm{~N}$ in the first circle plate bending tests), respectively. Substituting these material and geometric parameters into Eq. (21), one can obtain the relationship between critical cracking load $P_{c}$ and critical cracking radius $r_{c}=b$, as shown in Fig. 11, where the symbols are the experimental data, and the curves are modeling results based on Eq. (21). From Fig. 11, we find that the results of both the experimental measurement and the theoretical prediction for critical cracking load are consistent with each other for three coating-layerthickness cases. The critical cracking load increases with an increase in the radius of damage area; however, it decreases with an increase in coating layer thickness for a given radius of damage area.

\subsection{Interpretation to experimental failure phenomena based on the theoretical model}

In the subsection, we use the previously described theoretical model to interpret the experimental failure phenomena for both thin-coatinglayer case and thick-coating-layer case. When Eqs. (15) and (16) are combined, the ratio of circumferential bending moment to radial bending moment on the failure surface $\left(r=r_{c}=b\right)$ is given as follows:

$\left[\frac{M_{\theta}^{(2)}}{M_{r}^{(2)}}\right]_{c}=\frac{\frac{1-\eta}{b^{2}} c_{5}+[2(1+\eta) \ln b+(1+3 \eta)] c_{6}+2(1+\eta) c_{7}}{\frac{\eta-1}{b^{2}} c_{5}+[2(1+\eta) \ln b+(3+\eta)] c_{6}+2(1+\eta) c_{7}}$.

According to the fracture criterion based on the effective moment (Eq. (1)), the cracking direction on the failure surface is related to the ratio of bending moments. When the material and geometric parameters are substituted into Eq. (22), the change rule of the bending moment ratio with respect to the coating layer thickness can be obtained as shown in Fig. 12. The figure shows that the ratio of circumferential bending moment to radial bending moment increases with the increase in coating layer thickness and critical cracking radius. 


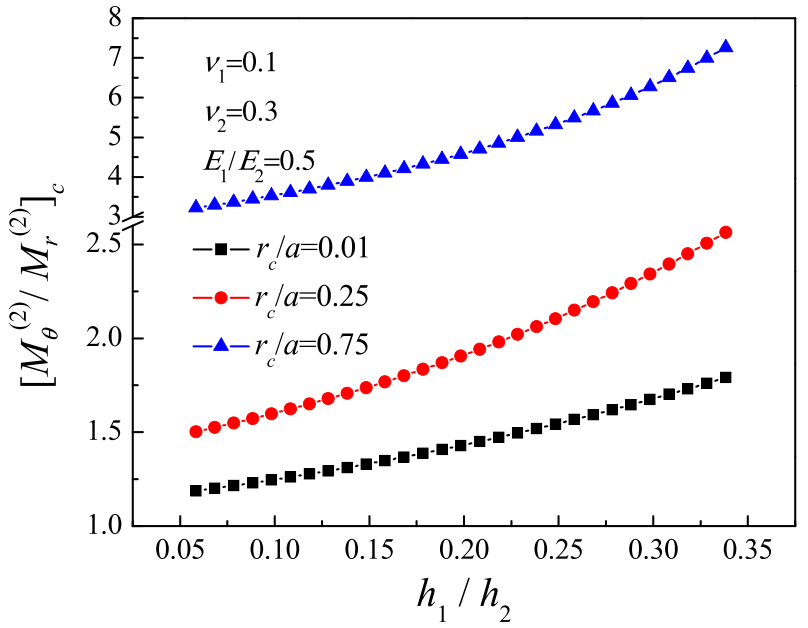

Fig. 12. The bending moment ratio on the failure surface versus dimensionless coating thickness for three dimensionless critical radius cases.

Specifically, the ratio increases with the increase in coating layer thickness. That is to say, for the coating layer fracture criterion based on the effective moment, the circumferential bending moment $M_{\ominus}$ plays from an important role as $M_{r}$ to a dominating role relative to $M_{r}$ with increasing coating layer thickness; therefore, with the increase in coating thickness, the fracture mode of the coating system changed from radial and ring cracking to mainly radial cracking, as shown in Fig. 8. For the thin coating systems (M1 and M2), the bending moment ratio was close to 1 near the center of the plate $\left(r_{c} / a=0.25\right)$, and therefore, both circumferential and radial bending moments played important roles, and radial and circumferential cracking occurred at the same time, as shown in Fig. 8(a) and Fig. 8(b). Meanwhile, the bending moment ratio is much bigger than $1\left(M_{\theta} / M_{r}=3.23\right.$ for $\mathrm{M} 1$ and $M_{\theta} / M_{r}=4.01$ for M2) far away from the center of the plate $\left(r_{c} / a=0.75\right)$, and therefore, the circumferential bending moment plays a dominant role, and mainly radial cracking occurred, as shown in Fig. 8(a) and Fig. 8(b). The theoretical model effectively explains the thickness-dependent fracture modes of coating systems observed in the experiments, as shown in Fig. 8. In addition, on the same failure surface, the influence of material parameters (Poisson's ratio and modulus of coating) on the variation of bending moment ratio with respect to coating thickness is discussed according to Eq. (22). Fig. 13 shows that the influence of Poisson's ratio on the change rule of the bending moment ratio with respect to coating thickness can be ignored. Fig. 14 shows that the

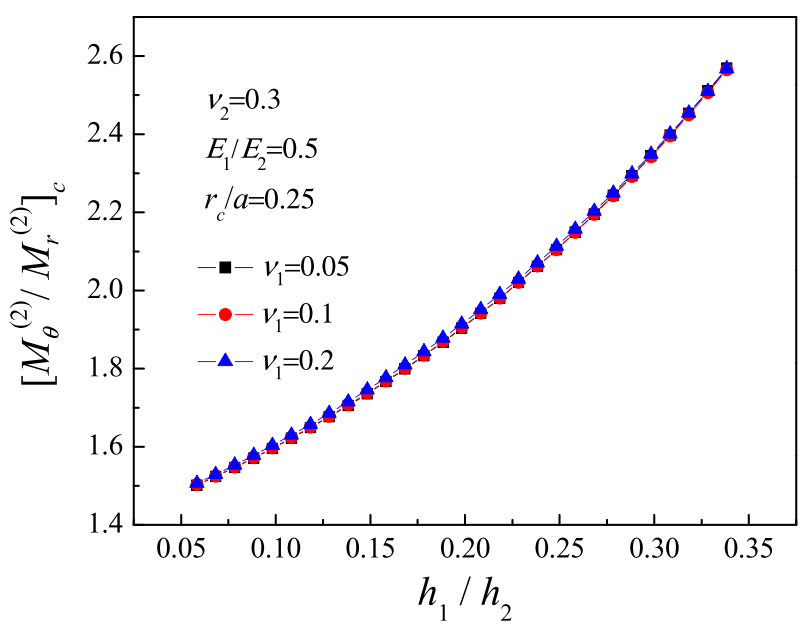

Fig. 13. The bending moment ratio on the failure surface $\left(r_{c} / a=0.25\right)$ versus dimensionless coating thickness for three Poisson's ratio cases.

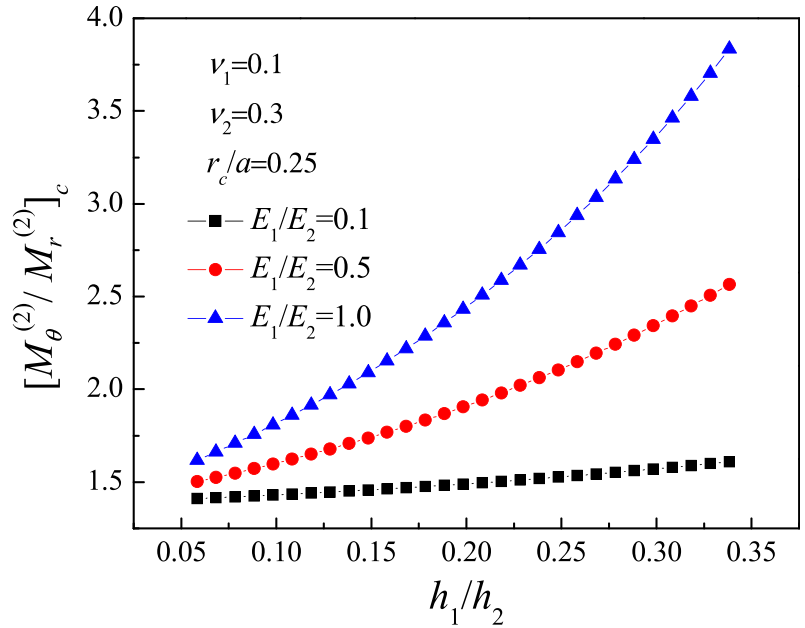

Fig. 14. The bending moment ratio on the failure surface $\left(r_{c} / a=0.25\right)$ versus dimensionless coating thickness for three modulus ratio cases.

bending moment ratio increases with the increase in coating modulus and that the influence of coating thickness on the bending moment ratio becomes more and more obvious with the increase in coating modulus.

\subsection{Damage and catastrophic failure characteristics based on the experimental measurements and the mathematical damage model}

Referring to literatures $[42,43]$, considering that a controlling variable $\lambda$ of one system is continuous and derivative for damage evolution $D$, we can use a Taylor expansion of the controlling variable at the catastrophic point $D_{f}$ to get

$\lambda(D)=\lambda_{f}+\left.\frac{\mathrm{d} \lambda(D)}{\mathrm{d} D}\right|_{D=D_{f}} *\left(D-D_{f}\right)+\left.\frac{1}{2} \frac{\mathrm{d} \lambda^{2}(D)}{\mathrm{d} D^{2}}\right|_{D=D_{f}} *\left(D-D_{f}\right)^{2}$.

In our experiment, the three kinds of coating systems were all fractured by tensile instability, so the normalized crack area is defined as the damage variable $D=A / A_{f}$. The normalized load $\lambda=P / P_{f}$ is taken as the controlling variable. $A$ is the summed area of all cracks, and the subscript $f$ represents the failure point of the coating. With $A=L * h_{1}$, the damage variable $D$ can be written as $D=L / L_{f}$, where $L$ corresponding to load $P$ is the summed length of all cracks. The failure load $P_{f}$ corresponds to the point where the length of cracks is saturated, as shown in Table 1 and Fig. 2. $D_{f}$ represents the complete damage corresponding to the normalized failure load $\lambda_{f}$. $D_{f}$ and $\lambda_{f}$ are both equal to 1 , based on the given definition. According to the catastrophic failure characteristics of brittle materials, the damage rate tends to be infinite at the catastrophic point, and thus $\lim _{\lambda \rightarrow \lambda_{f}} \frac{\mathrm{d} D}{\mathrm{~d} \lambda}=\infty$ and $\lim _{D \rightarrow D_{f}} \frac{\mathrm{d} \lambda}{\mathrm{d} D}=0$ can be obtained. When terms higher than the second order are neglected, and when it is combined with $\lim _{D \rightarrow D_{f}} \frac{\mathrm{d} \lambda}{\mathrm{d} D}=0$, Eq. (23) can be finally written as follows:

$D=1-C(1-\lambda)^{0.5}$,

where $C$ is the damage coefficient, $C=\left[\lambda^{\prime}\left(D_{f}\right) / 2\right]^{-0.5}$. Note that the initial damage $D_{0}=L_{0} / L_{f}$ of one sample is determined by the initial crack length $L_{0}$, corresponding to the initial load $P_{0}$ (the point where the cracks initiated, as shown in Table 1 and Fig. 2), and the corresponding normalized load is $\lambda_{0}=P_{0} / P_{f}$. Taking them into Eq. (24), we can acquire the damage coefficient $C=\frac{\left(1-D_{0}\right)}{\left(1-\lambda_{0}\right)^{0.5}}$. The initial damage $D_{0}$, initial normalized load $\lambda_{0}$, and damage coefficient $C$ based on the experiment measurements are shown in Table 1.

The damage rate of the coating system can be expressed by 


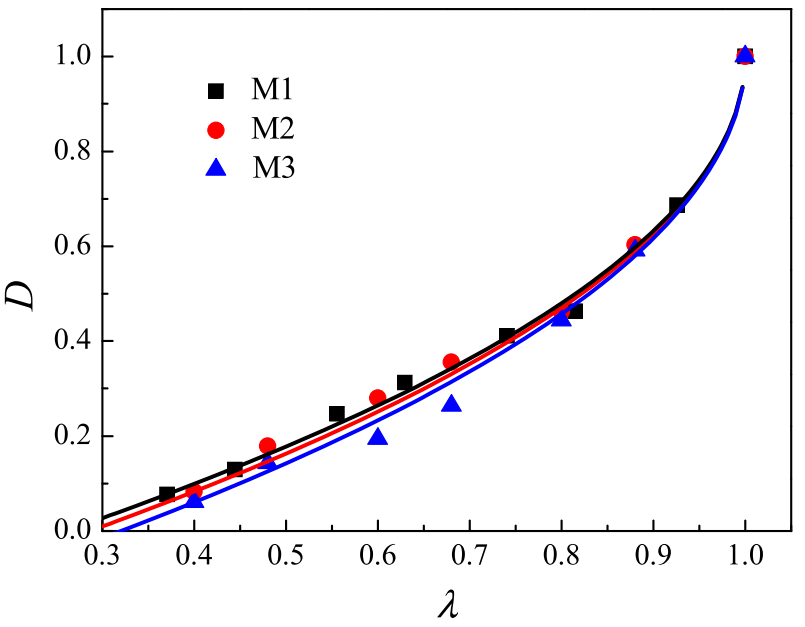

Fig. 15. Normalized damage versus normalized load for the three kinds of coating systems M1, M2, and M3 (circularly loaded to $3000 \mathrm{~N}$ in the first circle plate bending tests). The symbols are the experimental data, and the curves are based on Eq. (24).

$R=\frac{\mathrm{d} D}{\mathrm{~d} \lambda}=\frac{C}{2}(1-\lambda)^{-0.5}$.

Note that the mathematic damage model has been validated by experimental results $[42,43]$.

Fig. 15 shows that the experimentally measured damage evolution (symbols) for the samples M1, M2, and M3 (circularly loaded to $3000 \mathrm{~N}$ in the first circle plate bending tests) roughly corresponded with the power-law function in Eq. (24) (curves). The thicker the coating was, the faster the system was damaged. With the increase in coating thickness (from M1 to M3), the damage coefficient $C$ increased, as shown in Table 1 . The result agrees with the previous reports on threepoint bending [42] and four-point bending [43].

Fig. 16 shows the damage rate versus the normalized load of the three kinds of coating system based on the experimental data and Eq. (25). The damage rate of the coatings tended to become infinite as the load neared the failure load. Fig. 17 shows the logarithmic relation of the damage rate versus the normalized load. The slope of the curves is -0.5 , which indicates the power-law singularity of the damage rate. The intercept of the curves in Fig. 17 is equal to the average values of $\ln$ $(C / 2)$ of corresponding samples in terms of Eq. (25). It is reasonable to infer that the damage of the coating system sped up with the increase in

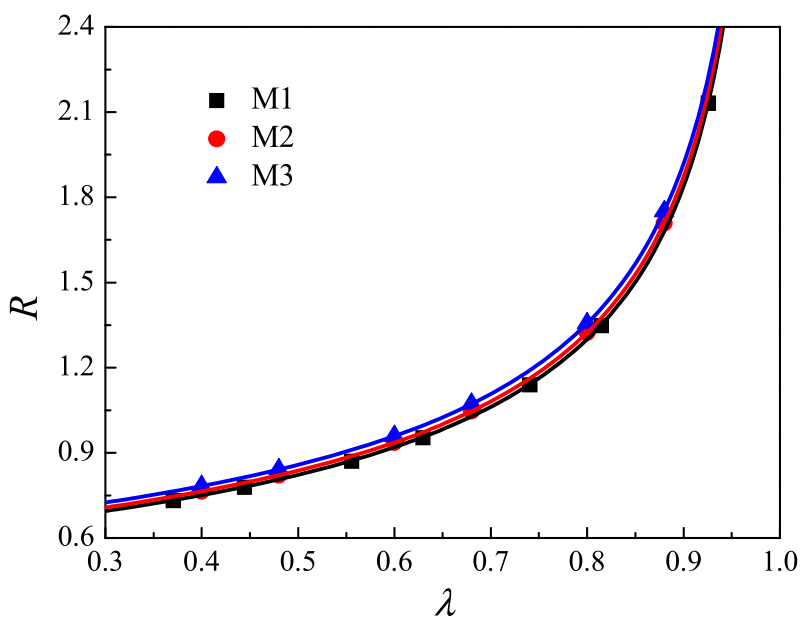

Fig. 16. Normalized damage rate versus normalized load for the three kinds of coating systems M1, M2, and M3 (circularly loaded to $3000 \mathrm{~N}$ in the first circle plate bending tests). The symbols are the experimental data, and the curves are based on Eq. (25).

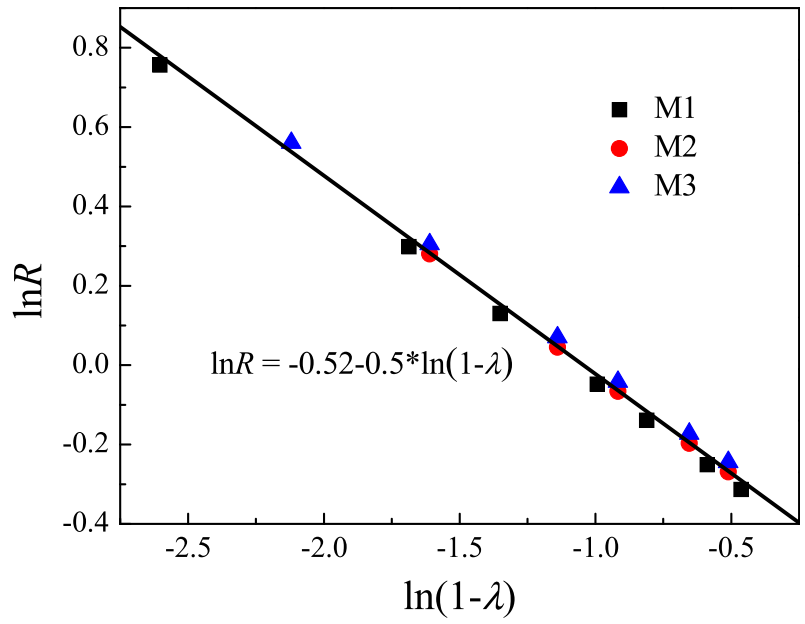

Fig. 17. Logarithmic relation of the damage rate versus the normalized load for the three kinds of coating systems M1, M2, and M3 (circularly loaded to $3000 \mathrm{~N}$ in the first circle plate bending tests). The symbols are the experimental data, and the curves denoted by the equation in the figure are based on the average values of the corresponding samples.

coating thickness, because radial cracking increasingly dominated the failure of system, whereas circumferential cracking is more local, and therefore less destructive, compared to the radial one.

\section{Conclusions}

Experimental observation and theoretical analysis were performed to study the damage and fracture characteristics of the three kinds of coating systems subjected to circle plate bending tests. The main conclusions can be summarized as follows:

(1) The fracture process demonstrated crucial three stages with a continuously applied load. The radical and circumferential cracks for the thin coating systems (mainly radical cracks for the thick coating system) were first initiated in the coating; they then propagated, multiplied, and became saturated.

(2) The three kinds of coating systems all fractured because of tensile instability. Radial and circumferential cracking dominated the systems for the thin coating systems, whereas mainly radial cracking dominated the systems for the thick ones. A theoretical model based on the effective moment concept was developed to explain the thickness-dependent fracture characteristics of the coating systems. Moreover, the model can effectively predict the variation of the critical cracking load with respect to the critical cracking radius.

(3) The mathematic damage model could effectively describe the damage evolution behaviors of the coating systems. The damage rate shows a power-law singularity, and the thick coating system were damaged quickly than the thin ones. The experimental measurements for the three kinds of coatings were all consistent with predictions based on the model.

\section{Declaration of competing interest}

The authors declare that they have no known competing financial interests or personal relationships that could have appeared to influence the work reported in this paper.

\section{Acknowledgment}

This work was supported by the National Natural Science Foundation of China, China grants of China (Nos. 11890681, 


\section{Appendix A. The expressions of coefficients $c_{1}-c_{8}$ in deflection functions}

$c_{1}=0$

$c_{2}=\frac{P}{8 \pi S_{11}^{(1)}}$

$c_{3}=\frac{c_{2}}{2 \mathrm{~A}}\left\{\begin{array}{l}-2 b^{2} \ln (b)+\left(a^{2}-b^{2}\right) 2 \ln (b) \nu_{2} \zeta \eta+\left(a^{2}-b^{2}\right)+2 b^{2} \ln (b) \zeta(1-\eta) \\ +2 \ln (b) \nu_{2} \zeta\left(a^{2}+b^{2}\right)+v_{2} \zeta \eta\left(a^{2}-b^{2}\right)-2 \ln (b) a^{2} \zeta \eta \\ +2[2(1+\eta) \ln (a)+\eta+3] \zeta a^{2}+\zeta b^{2}-b^{2} \eta \zeta+\left(a^{2}+b^{2}\right) \nu_{2} \zeta-2 a^{2} \ln (b) \zeta \\ -a^{2} \eta \zeta-2 a^{2} \ln (b) \eta^{2}+2 b^{2} \ln (b) \eta^{2}-a^{2} \zeta+2 a^{2} \ln (b)-a^{2} \eta^{2}+b^{2} \eta^{2}\end{array}\right\}$

$c_{4}=-\frac{c_{2}}{2 \mathrm{~A}}\left\{\begin{array}{l}-a^{2} b^{2} \nu_{2} \eta \zeta^{2}+a^{2} b^{2} \nu_{2} \eta \zeta+b^{4}\left(\nu_{2} \eta \zeta^{2}-\nu_{2} \eta \zeta\right)+a^{2} b^{2}\left(\nu_{2} \zeta^{2}+\nu_{2} \zeta-\eta \zeta+\eta^{2} \zeta-\eta \zeta^{2}\right) \\ +[2(1+\eta) \ln (a)+\eta+3] a^{4} \nu_{2} \zeta^{2}+2 \ln (a) a^{4}\left(\eta^{2} \zeta-\eta \zeta^{2}-\nu_{2} \zeta^{2}\right)-4 \ln (b)^{2} a^{2} b^{2} \zeta^{2} \\ -[2(1+\eta) \ln (a)+\eta+3]\left(a^{4} \eta \zeta+a^{2} b^{2} \zeta^{2}\right)+4 \ln (b)^{2} a^{2} b^{2} \zeta+2 \ln (b) a^{2} b^{2} \zeta^{2}+ \\ {[2(1+\eta) \ln (a)+\eta+3] a^{2} b^{2} \zeta-2 \ln (b) a^{2} b^{2} \zeta+a^{2} b^{2}+b^{4} \eta^{2}+2 b^{4} \zeta-b^{4} \zeta^{2}-b^{4}} \\ -4 \ln (b)^{2} a^{2} b^{2} \eta \nu_{2} \zeta^{2}-2[2(1+\eta) \ln (a)+\eta+3] a^{2} b^{2} \nu_{2} \zeta^{2}(\ln (b)-\ln (a)) \\ -4 \ln (a) \ln (b) a^{2} b^{2}\left(\eta^{2} \zeta-\eta \zeta^{2}-\nu_{2} \zeta^{2}\right)+[4 \ln (a)-2 \ln (b)] a^{2} b^{2} \eta \nu_{2} \zeta^{2}+ \\ -2[2(1+\eta) \ln (a)+\eta+3] a^{2} b^{2} \eta \zeta[\ln (a)-\ln (b)]-8 \ln (a) \ln (b) a^{2} b^{2} \eta \zeta \\ -b^{4}\left(\nu_{2} \zeta^{2}-\nu_{2} \zeta+\eta \zeta+\eta^{2} \zeta-\eta \zeta^{2}\right)-a^{2} b^{2}\left(\eta^{2}+2 \zeta-\zeta^{2}\right)+[2(1+\eta) \ln (a)+\eta+3] \\ \left(a^{4} \zeta^{2}+a^{4} \zeta\right)-2 \ln (a)\left(a^{4} \zeta^{2}+a^{4} \zeta\right)+4 \ln (a) \ln (b) a^{2} b^{2} \eta \nu_{2} \zeta^{2}-2 \ln (a) a^{4} \eta \nu_{2} \zeta^{2} \\ +4 \ln (b)^{2} a^{2} b^{2}\left(\eta^{2} \zeta-\eta \zeta^{2}-\nu_{2} \zeta^{2}\right)+[2(1+\eta) \ln (a)+\eta+3] a^{2} b^{2} \zeta^{2}\left[2 \ln (b)-\nu_{2}\right] \\ +4 a^{2} b^{2} \ln (a)\left[\ln (b) \zeta^{2}-\eta^{2} \zeta+\eta \zeta^{2}\right]+2 a^{2} b^{2} \ln (b)\left[4 \ln (b) \eta \zeta+\eta^{2} \zeta-\eta \zeta^{2}+\nu_{2} \zeta^{2}\right] \\ -[2(1+\eta) \ln (a)+\eta+3] a^{2} b^{2}[2 \zeta-\eta \zeta]-4 a^{2} b^{2} \ln (a)[\ln (b) \zeta+\eta \zeta]\end{array}\right\}$

$c_{5}=\frac{c_{2} b^{2} \zeta a^{2}}{\mathrm{~A}}\left\{\begin{array}{l}{\left[2 \ln (b) \eta \nu_{2} \zeta-(2(1+\eta) \ln (a)+\eta+3) \nu_{2} \zeta\right]} \\ -2 \ln (b) \eta^{2}+2 \ln (b) \nu_{2} \zeta+\eta \nu_{2} \zeta+2[2(1+\eta) \ln (a)+\eta+3] \eta \\ -[2(1+\eta) \ln (a)+\eta+3] \zeta-4 \ln (b) \eta+2 \ln (b) \zeta-\eta^{2} \\ +\eta \zeta+\nu_{2} \zeta+[2(1+\eta) \ln (a)+\eta+3]-2 \ln (b)+\zeta-1\end{array}\right\}$

$c_{6}=\frac{P}{8 \pi S_{11}^{(2)}}$

$c_{7}=-\frac{c_{2} \zeta}{2 \mathrm{~A}}\left\{\begin{array}{l}2 \ln (b) \nu_{2} \zeta b^{2} \eta-[2(1+\eta) \ln (a)+\eta+3] a^{2} \nu_{2} \zeta-2 \ln (b) b^{2}\left(\eta^{2}-\eta \zeta+\nu_{2} \zeta\right) \\ +\nu_{2} \zeta b^{2} \eta+[2(1+\eta) \ln (a)+\eta+3] a^{2}(\eta-\zeta-1)-2 \ln (b) b^{2} \zeta-b^{2} \eta^{2}+b^{2} \eta \zeta \\ -b^{2} \nu_{2} \zeta+2 \ln (b) b^{2}-b^{2} \zeta+b^{2}\end{array}\right\}$

$c_{8}=-\frac{\zeta a^{2} c_{2}}{2 \mathrm{~A}}\left\{\begin{array}{l}2 \ln (a) a^{2} \eta^{2}-4 \ln (a) b^{2} \eta^{2}+2[2(1+\eta) \ln (a)+\eta+3] \ln (a) b^{2}-4 \ln (a) \ln (b) b^{2} \\ -4 \ln (a) b^{2} \eta-2 \ln (b) b^{2}-2 \ln (b) b^{2} \nu_{2} \zeta \eta-b^{2}-2 a^{2} \ln (a) \zeta+2 b^{2} \ln (b) \zeta \\ -2 b^{2} \ln (b) \eta \zeta+2 b^{2} \ln (b) \nu_{2} \zeta-b^{2} \nu_{2} \zeta \eta+[2(1+\eta) \ln (a)+\eta+3] a^{2} \zeta+b^{2} \zeta \\ -b^{2} \eta \zeta+b^{2} \nu_{2} \zeta+2 \ln (b) b^{2} \eta^{2}+b^{2} \eta^{2}+4 \ln (a) \ln (b) b^{2} \nu_{2} \zeta \eta \\ -[2(1+\eta) \ln (a)+\eta+3] a^{2}(\eta-1)-2 \ln (a) a^{2}-2[2(1+\eta) \ln (a)+\eta+3] \ln (a) b^{2} \nu_{2} \zeta \\ +4 \ln (a) \ln (b) b^{2} \eta \zeta+4 \ln (a) \ln (b) b^{2} \nu_{2} \zeta-2 a^{2} \ln (a) \nu_{2} \zeta \eta+4 b^{2} \ln (a) \nu_{2} \zeta \eta \\ +[2(1+\eta) \ln (a)+\eta+3] a^{2} \nu_{2} \zeta+[2(1+\eta) \ln (a)+\eta+3] \ln (a) b^{2}(\eta-2 \zeta) \\ -4 \ln (a) \ln (b) b^{2} \eta^{2}-8 \ln (a) \ln (b) b^{2} \eta+4 \ln (a) \ln (b) b^{2} \zeta-2 a^{2} \ln (a) \nu_{2} \zeta+4 \ln (a) b^{2} \eta \zeta\end{array}\right\}$

where $\mathrm{A}=b^{2}\left(\nu_{2} \zeta \eta-\eta^{2}+\zeta \eta-\nu_{2} \zeta-\zeta+1\right)-a^{2}\left(\nu_{2} \zeta \eta-\eta^{2}+\zeta \eta+\nu_{2} \zeta+\zeta+1\right), \zeta=\frac{s_{11}^{(1)}}{S_{11}^{(2)}}, \eta=\frac{S_{12}^{(2)}}{S_{11^{(2)}}}$.

\section{References}

[1] T.E. Strangman, Thermal barrier coatings for turbine airfoils, Thin Solid Films 127 (1985) 93-106.

[2] S. Bose, J. Demasimarcin, Thermal barrier coating experience in gas turbine engines at Pratt \& Whitney, J. Therm. Spray Technol. 6 (1997) 99-104.

[3] R.E. Taylor, X.W. Wang, X.F. Xu, Thermophysical properties of thermal barrier coatings, Surf. Coat. Technol. 120 (1999) 89-95.

[4] N.P. Padture, M. Gell, E.H. Jordan, Thermal barrier coatings for gas-turbine engine applications, Science 296 (2002) 280-284.

[5] N. Curry, N. Markocsan, X.H. Li, A. Tricoire, M.R. Dorfman, Next generation thermal barrier coatings for the gas turbine industry, J. Therm. Spray Technol. 20 (2011) 108-115.
[6] A.M. Freborg, B.L. Ferguson, W.J. Brindley, G.J. Petrus, Modeling oxidation induced stresses in thermal barrier coatings, Mater. Sci. Eng. A 245 (1998) 182-190.

[7] S. Ahmadian, C. Thistle, E.H. Jordan, Experimental and finite element study of an air plasma sprayed thermal barrier coating under fixed cycle duration at various temperatures, J. Am. Ceram. Soc. 96 (2013) 3210-3217.

[8] Y. Bai, C.H. Ding, H.Q. Li, Z.H. Han, B.J. Ding, T.J. Wang, L. Yu, Isothermal oxidation behavior of supersonic atmospheric plasma-sprayed thermal barrier coating system, J. Therm. Spray Technol. 22 (2013) 1201-1209.

[9] S. Ahmadian, E.H. Jordan, Explanation of the effect of rapid cycling on oxidation, rumpling, microcracking and lifetime of air plasma sprayed thermal barrier coatings, Surf. Coat. Technol. 244 (2014) 109-116.

[10] S. Rangaraj, K. Kokini, Estimating the fracture resistance of functionally graded thermal barrier coatings from thermal shock tests, Surf. Coat. Technol. 173 (2003) 201-212. 
[11] W.Q. Wang, C.K. Sha, D.Q. Sun, X.Y. Gu, Microstructural feature, thermal shock resistance and isothermal oxidation resistance of nanostructured zirconia coating, Mater. Sci. Eng. A 424 (2006) 1-5.

[12] G.D. Girolamo, F. Marra, C. Blasi, E. Serra, T. Valente, Microstructure, mechanical properties and thermal shock resistance of plasma sprayed nanostructured zirconia coatings, Ceram. Int. 37 (2011) 2711-2717.

[13] N.A. Fleck, A.C.F. Cocks, S. Lampenscherf, Thermal shock resistance of air plasma sprayed thermal barrier coatings, J. Eur. Ceram. Soc. 34 (2014) 2687-2694.

[14] D.M. Yang, Y. Gao, H.J. Liu, C.Q. Sun, Thermal shock resistance of bimodal structured thermal barrier coatings by atmospheric plasma spraying using nanostructured partially stabilized zirconia, Surf. Coat. Technol. 315 (2017) 9-16.

[15] Y.C. Zhou, T. Tonomori, A. Yoshida, L. Liu, G. Bignall, T. Hashida, Fracture char acteristics of thermal barrier coatings after tensile and bending tests, Surf. Coat. Technol. 157 (2002) 118-127.

[16] L.H. Yang, Y.C. Zhou, W.G. Mao, C.S. Lu, Real-time acoustic emission testing based on wavelet transform for the failure process of thermal barrier coatings, Appl. Phys. Lett. 93 (2008) 231906.

[17] Z.B. Chen, Z.G. Wang, S.J. Zhu, Tensile fracture behavior of thermal barrier coatings on superalloy, Surf. Coat. Technol. 205 (2011) 3931-3938.

[18] L. Wang, C.G. Liu, X.H. Zhong, Y.X. Zhao, H.Y. Zhao, J.S. Yang, S.Y. Tao, Y. Wang, Investigation of crack propagation behavior of atmospheric plasma-sprayed thermal barrier coatings under uniaxial tension using the acoustic emission technique, J. Therm. Spray Technol. 24 (2015) 296-308.

[19] A.K. Ray, R.W. Steinbrech, Crack propagation studies of thermal barrier coatings under bending, J. Eur. Ceram. Soc. 19 (1999) 2097-2109.

[20] Y. Yamazaki, A. Schmidt, A. Scholz, The determination of the delamination resistance in thermal barrier coating system by four-point bending tests, Surf. Coat. Technol. 201 (2006) 744-754.

[21] Z.X. Chen, Z.G. Wang, F.H. Yuan, S.J. Zhu, Interfacial fracture behavior of a thermal barrier coating system under four-point bend loading, Mater. Sci. Eng. A 483 (2008) 629-632.

[22] P.F. Zhao, X.D. Li, F.L. Shang, C.J. Li, Interlamellar cracking of thermal barrier coatings with TGOs by non-standard four-point bending tests, Mater. Sci. Eng. A 528 (2011) 7641-7647.

[23] X.N. Li, L.H. Liang, J.J. Xie, L. Chen, Y.G. Wei, Thickness-dependent fracture characteristics of ceramic coatings bonded on the alloy substrates, Surf. Coat. Technol. 258 (2014) 1039-1047.

[24] H.Y. Liu, Y.G. Wei, L.H. Liang, X.H. Liu, Y.B. Wang, H.S. Ma, Damage characterization model of ceramic coating systems based on energy analysis and bending tests, Ceram. Int. 44 (2018) 4807-4813.

[25] H. Chai, B.R. Lawn, Fracture mode transitions in brittle coatings on compliant substrates as a function of thickness, J. Mater. Res. 19 (2004) 1752-1761.

[26] C. He, Z.Q. Xie, Z.B. Guo, H.M. Yao, Fracture-mode map of brittle coatings: theoretical development and experimental verification, J. Mech. Phys. Solids 83 (2015) 19-35.

[27] W.G. Mao, J. Wan, C.Y. Dai, J. Ding, Y. Zhang, Y.C. Zhou, C. Lu, Evaluation of microhardness, fracture toughness and residual stress in a thermal barrier coating system: a modified Vickers indentation technique, Surf. Coat. Technol. 206 (2012) 4455-4461 https://www.sciencedirect.com/science/article/abs/pii/ S0257897212003908-!.

[28] Z.L. Qu, K. Wei, Q. He, R.J. He, Y.M. Pei, S.X. Wang, D.N. Fang, High temperature fracture toughness and residual stress in thermal barrier coatings evaluated by an in-situ indentation method, Ceram. Int. 44 (2018) 7926-7929.

[29] M. Białas, P. Majerus, R. Herzog, Z. Mróz, Numerical simulation of segmentation cracking in thermal barrier coatings by means of cohesive zone elements, Mater. Sci. Eng. A 412 (2005) 241-251.

[30] W. Zhu, L. Yang, J.W. Guo, Y.C. Zhou, C. Lu, Numerical study on interaction of surface cracking and interfacial delamination in thermal barrier coatings under tension, Appl. Surf. Sci. 315 (2014) 292-298.

[31] R. Xu, X.L. Fan, T.J. Wang, Mechanisms governing the interfacial delamination of thermal barrier coating system with double ceramic layers, Appl. Surf. Sci. 370 (2016) 394-402.

[32] W.G. Mao, C.Y. Dai, L. Yang, Y.C. Zhou, Interfacial fracture characteristic and crack propagation of thermal barrier coatings under tensile conditions at elevated temperatures, Int. J. Fract. 151 (2008) 107-120.

[33] X.C. Zhang, M. Watanabe, S. Kuroda, Effects of residual stress on the mechanical properties of plasma-sprayed thermal barrier coatings, Eng. Fract. Mech. 110 (2013) 314-327.

[34] M. Zhou, W.B. Yao, X.S. Yang, Z.B. Peng, K.K. Li, C.Y. Dai, W.G. Mao, Y.C. Zhou, C. Lu, In-situ and real-time tests on the damage evolution and fracture of thermal barrier coatings under tension: a coupled acoustic emission and digital image correlation method, Surf. Coat. Technol. 240 (2014) 40-47.

[35] M. Schweda, T. Beck, J. Malzbender, L. Singheiser, Damage evolution of a therma barrier coating system with 3-dimensional periodic interface roughness: effects of roughness depth, substrate creep strength and pre-oxidation, Surf. Coat. Technol. 276 (2015) 368-373.

[36] L.L. Wang, Q.B. Fan, Y.B. Liu, G.J. Li, H.M. Zhang, Q.S. Wang, F.C. Wang, Simulation of damage and failure processes of thermal barrier coatings subjected to a uniaxial tensile load, Mater. Des. 86 (2015) 89-97.

[37] L.H. Qian, S.J. Zhu, Y. Kagawa, T. Kubo, Tensile damage evolution behavior in plasma-sprayed thermal barrier coating system, Surf. Coat. Technol. 173 (2003) $178-184$.

[38] A.P. McGuigan, G.A.D. Briggs, V.M. Burlakov, M. Yanaka, Y. Tsukahara, An elastic-plastic shear lag model for fracture of layered coatings, Thin Solid Films 424 (2003) 219-223.

[39] T.S. Hille, S. Turteltaub, A.S.J. Suiker, Oxide growth and damage evolution in thermal barrier coatings, Eng. Fract. Mech. 78 (2011) 2139-2152.

[40] L. Yang, Z.C. Zhong, Y.C. Zhou, C.S. Lu, Quantitative assessment of the surface crack density in thermal barrier coatings, Acta Mech. Sin. 30 (2014) 167-174.

[41] L. Saucedo-Mora, K. Slámečka, U. Thandavamoorthy, T.J. Marrow, Multi-scale modeling of damage development in a thermal barrier coating, Surf. Coat. Technol 276 (2015) 399-407.

[42] L.H. Liang, X.N. Li, H.Y. Liu, Y.B. Wang, Y.G. Wei, Power-law characteristics of damage and failure of ceramic coating systems under three-point bending, Surf Coat. Technol. 285 (2016) 113-119.

[43] H.Y. Liu, L.H. Liang, Y.B. Wang, Y.G. Wei, Fracture characteristics and damage evolution of coating systems under four-point bending, Int. J. Appl. Ceram. Technol. 13 (2016) 1043-1052.

[44] S.W. Hao, F. Rong, M.F. Lu, H.Y. Wang, M.F. Xia, F.J. Ke, Y.L. Bai, Power-law singularity as a possible catastrophe warning observed in rock experiments, Int. J. Rock Mech. Min. Sci. 60 (2013) 253-262.

[45] L.H. Liang, H. Wei, X.N. Li, Y.G. Wei, Size-dependent interface adhesive energy and interface strength of nanostructured systems, Surf. Coat. Technol. 236 (2013) 525-530.

[46] L. Wang, Y. Wang, W.Q. Zhang, X.G. Sun, J.Q. He, Z.Y. Pan, Finite element simulation of stress distribution and development in 8YSZ and double-ceramic-layer $\mathrm{La}_{2} \mathrm{Zr}_{2} \mathrm{O}_{7} / 8 \mathrm{YSZ}$ thermal barrier coatings during thermal shock, Appl. Surf. Sci. 258 (2012) 3540-3551. 